\title{
Guest Editors' Introduction to the Special Issue on Knowledge-Driven Business Process Management
}

\author{
ADITYA GHOSE, University of Wollongong, Australia \\ HAMID R. MOTAHARI NEZHAD, University of New South Wales, Australia \\ MANFRED REICHERT, University of Ulm, Germany
}

Business Process Management (BPM) has traditionally focused on well-structured and formally designed and executed business processes. Several business processes, however, are knowledgecentric, defined dynamically by knowledge workers, or creative (i.e., covering a unique situation). We call this larger class of processes "knowledge-driven".

We anticipate that knowledge-driven BPM systems will support human decision making and devices in IoT, which requires leveraging knowledge about processes and their contexts in an automated and proactive manner. A variety of knowledge is relevant in such settings, e.g., concerning process activities, causal relationships, process goals, and enterprise architectures. Knowledgedriven BPM can involve novel conceptions of process specification (e.g. using commitments or strategy models) or the extraction of process knowledge from available artifacts as well as novel process analysis techniques leveraging richer knowledge about processes.

This special section sought to identify the best and brightest proposals in this emerging, broadly defined space. A total of 5 submissions were accepted from 19 submitted papers.

Adaptive Case Management (ACM) represents a prominent (and particularly interesting) approach to the management of knowledge-driven processes. The article by Eshuis, Hull and Yi titled "Reasoning about Property Preservation in Adaptive Case Management" makes important contributions to the understanding of change management in ACM systems. They offer the means to ensure that ACM systems (specifically under the Guard-Stage-Milestone model) that have been carefully designed to satisfy a set of (desirable) properties continue to do so when subjected to change (a business reality for most modern process management systems). The authors subject the problem to a careful formal analysis and also argue that the resulting framework is effective in reasoning about rollbacks.

The article by Armas-Cervantes, Dumas, La Rosa and Maaradji titled "Local Concurrency Detection in Business Process Event Logs" addresses a problem in process discovery that is particularly acute in the instance of knowledge-driven processes. The problem has to do with the detection of concurrency when mining process/event logs. Traditional approaches to process discovery seek to make global statements about the concurrency of certain event types, thus implying that all instances of these event types must be concurrent. In many knowledge-driven processes, this is not the case. The authors offer a more nuanced approach, where concurrency relations are

Authors' addresses: A. Ghose, School of Computing and Information Technology, University of Wollongong, NSW 2522 Australia; email: aditya@uow.edu.au; H. R. Motahari Nezhad, University of New South Wales, 550 High St Palo Alto, CA 94304; email: hamidm@cse.unsw.edu.au; M. Reichert, University of Ulm, Germany.

Permission to make digital or hard copies of part or all of this work for personal or classroom use is granted without fee provided that copies are not made or distributed for profit or commercial advantage and that copies bear this notice and the full citation on the first page. Copyrights for third-party components of this work must be honored. For all other uses, contact the owner/author(s).

(C) 2019 Copyright held by the owner/author(s).

1533-5399/2019/02-ART11

https://doi.org/10.1145/3296981 
more local in nature, being applicable only when certain conditions are true, or relative to a specific set of states.

It is important to measure the performance of knowledge-intensive processes, recognizing that specialized measures may be required (relative to those used for structured processes). The article by Estrada-Torres, Richetti, del-Rio-Ortega, Baiao, Resinas, Santoro, and Cortes titled "Measuring Performance in Knowledge Intensive Processes" considers this problem in some detail. They offer a novel ontology for these specialized process performance indicators. They also provide a methodology (leveraging notions of lead and lag indicators) to assist in the execution of knowledgeintensive processes in ways that would achieve specified performance targets.

The problem of devising a reference architecture for business process management systems that would be amenable to knowledge-driven processes is addressed by Pourmirza, Peters, Dijkman and Grefen in their article entitled "BPMS-RA: A Novel Reference Architecture for Business Process Management Systems.” Their BPMS-RA reference architecture makes special provisions for business process analytics, data-driven process execution, and a range of other requirements for knowledge-driven processes.

Finally, the article by Yousfi, Batouli, and Weske titled "Achieving Business Process Improvement via Ubiquitous Decision-Aware Business Processes” addresses the critical questions of decision awareness and ubiquity in business process models. Ubiquitous processes permit us to address the relatively less-understood domain of business processes interacting with physical infrastructures (such as IoT systems). Decision-aware processes permit us to bring to bear carefully crafted bodies of knowledge (for instance, in the form of decision tables). The authors illustrate these concepts through a detailed case study.

As with any collection such as this, the articles in this issue do not by any means represent the final word on knowledge-driven business processes. A vast repertoire of open questions remain, to be addressed by the community-hopefully in the near future. 